\title{
Forever Blowing Bubbles
}

Benoit Dubertret and Sabine Loüet report on developments in the world of foams, as seen through the eyes of the participants at the second Foams Euroconference.

Some 150 scientists from 15 countries met to present their findings at the second Foams Euroconference held in Arcachon, near Bordeaux, on May 28 - June 2. It was chaired by Dominique Langevin of the Centre Paul Pascal (Bordeaux). Thanks to European Union funding, scientists from the European Community and countries such as Bulgaria, Russia, Brazil, Israel, China, Switzerland, and the USA, joined in this effervescence of foam science.

Some of the discussions centred on the century-old ideas of Lord Kelvin. While Archimedes is often portrayed in his bath, Kelvin should be shown in a bubble bath! In 1887 , he conjectured that the ether was a liquid foam. This mysterious substance was then considered the essential medium for electromagnetic waves to propagate. Kelvin's ingenuity hinted at the prominent role foam plays in our surrounding world. In the 19th century, Stokes, Green, and Plateau paved the way for the description of foams that Smith and others went on to elaborate in the 2oth century. The 1992 Nobel Prize winner P.G. de Gennes concluded his acceptance speech with the words "amusons-nous, [...] tout n'est que bulles de savon." He was underlining the importance of foams (ether is no longer fashionable) which provide a fascinating example of soft matter with considerable industrial interest.

Today, the study of foams is pursued in research areas ranging from physics (stimulated by an article of Weaire and Rivier published in Contemporary Physics in 1984) to mathematics, chemistry and engineering. During the conference, three different levels of study were presented: - On the microscopic scale $(1 \mathrm{~nm})$ research focuses on the effect of surfactants (often mixed with polymers) and of anti-foaming agents.

- On the mesoscopic scale $(1 \mu \mathrm{m})$, the elasticity, viscosity and phenomena of local drainage (disappearance of inter-bubble liquid) in individual thin films are studied. - On the macroscopic scale (1 $\mathrm{mm}$ or more) foams properties are characterized

Benoit Dubertret is a Ph.D. student in Strasbourg working on biological foams.

Sabine Loüet recently completed a M.Sc. in science communication at Imperial College, London. by means of mathematical tools such as statistics, topology, geometry and continuum theory, in a variety of ways.

The microscopic aspects are related to industrial problems of foaming control and soap-film stability. Foaming which is too abundant or long-lasting can be a nuisance. On the other hand, suitable foam gives bread an enjoyable texture. Latest results suggest that it is crucial to understand the phase of the polymer - surfactant mixture added to the foam and the molecular structure of its constituents to explain a foam's stability or degradation.

The mesoscopic aspect attracted much more attention than in the first Euroconference held in Renvyle, Ireland, in 1994. Scientists understand better how film equilibrium (and thus the stability of a foam) depends on the forces acting in the liquid trapped in between the bubbles. They also begin to understand how stability is influenced by the combined effects of drainage and gas diffusion between the foam bubbles, and hence how degradation occurs in foams.

At the macroscopic level, theory often takes a statistical point of view, considering foam topology and geometry. The models (dynamics and stability of random cellular structures) prove remarkably successful in two dimensions, and can even be applied to biological tissues such as the human skin. The three-dimensional models, more complex, require powerful computer simulations. However, with advanced software, different models can be tested and the micromechanics of complicated structures studied.

Experimental and theoretical work attempts to solve the problems of foam rheology and drainage (reactions and local transformations under different stress and
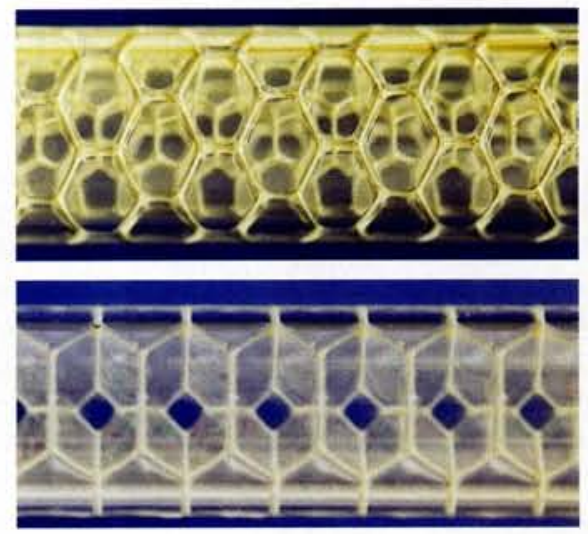

Two different crystalline structures of a soap froth with bubbles of equal size in a cylindrical tube.

strain; influence and control of drainage). These questions are not as arcane as they may seem at first sight. For example, foam scientists try to understand phenomena such as elasticity and the flow involved when a head of beer is formed, or when shaving foam spreads on the face. The process taking place when a meringue is baked also involves interesting mechanisms. The mechanical properties of solid foams, and how foam flows in a porous medium are also of interest. The strong Russian tradition in the area of acoustic and seismic wave propagation in foams was well represented by different presentations. Further applications are found in the petroleum and building industries.

A quick survey among participants showed their general satisfaction with both the scientific content and the organization. A few expressed interest in solid foam and fluid dynamics, areas to be developed in the future. One concluded "Let's hope that the exploration of the world of foams, although at its embryonic stage, will develop further through stimulating initiatives like the second Foams Euroconference." Once as mysterious as the ether itself, foams lose little of their fascination as their properties finally come within our grasp. A third Foams Euroconference is planned for 1998 , probably in Portugal.

\section{Mix, Stir and Serve - An Instant Recipe from Bordeaux}

The celebrated speculations of Kelvin were concerned with the ideal structure of a foam of bubbles of uniform size, tightly compressed together. Such a monodisperse foam still attracts the attention of physicists, in their search for simplicity. In one of the highlights of the Arcachon conference, Jeröme Bibette described a new technique for making monodisperse emulsions. An emulsion is a two-phase liquid system, closely analogous to a foam, and often much more stable. Bibette and his colleagues at the Centre Pascal in Bordeaux offer the following recipe. Prepare a coarse emulsion, much as one makes a French Dressing for salad. Then submit it to rapid shearing motion, et voila, it breaks up into identical droplets. As with all food recipes, the secret lies in the ingredients, which have special viscoelastic properties. The mechanism of formation is not fully explained, but that will not stop experimentalists adopting this convenient trick. The work of Bibette echoes that of the Nobel Laureate Jean Perrin, who described how to make monodisperse emulsions in his classic work Les Atomes, three-quarters of a century ago. But Perrin's method, and those which followed were laborious. Now we have an instant recipe.

D.L. Weaire 